\title{
Heuristic model for the power threshold of the L-H transition
}

\author{
R. Bilato, C. Angioni, G. Birkenmeier, F. Ryter and \\ ASDEX Upgrade Team ${ }^{\dagger}$ \\ Max Planck Institute for Plasma Physics, EURATOM Association, \\ Boltzmannstr. 2, 85748 Garching, Germany \\ ( ${ }^{\dagger}$ See the author list of H. Meyer et al., Nucl. Fusion 59 (2019), 112014) \\ E-mail: roberto(dot)bilato (at) ipp (dot) $\mathrm{mpg}($ dot $)$ de
}

\begin{abstract}
Guided by the recent experimental finding of the key role played by the ion heat channel in the transition from low $(\mathrm{L})$ to high $(\mathrm{H})$ confinement mode [F. Ryter et al, Nuclear Fusion 54 (2014) 083003, M. Schmidtmayr et al, Nuclear Fusion 58 (2018) 056003], the present phenomenological model of the power threshold for the L-H transition is developed by combining the energy equipartition between electrons and ions, and the L-mode scaling of the energy confinement time. This heuristic model describes with continuity the transition of the power threshold from the low- to the high-density branches and clarifies the origin of the main dependence of the power threshold on the plasma current in the low density branch.
\end{abstract}

PACS numbers: 


\section{Introduction}

When the auxiliary heating power in a divertor fusion device is increased above a certain threshold, $P_{\mathrm{L}-\mathrm{H}}$, the energy and particle confinement experiences a sudden improvement with a clear bifurcation of the confinement regime, from the so-called Lmode to H-mode confinement [1]. The signature of the H-mode is the formation of an edge-transport-barrier (ETB), characterized by a pedestal-like increase of the plasma temperature and density nearby the last-closed magnetic surface. In experiments it has been observed that $P_{\mathrm{L}-\mathrm{H}}$ is not monotonic with the line-averaged plasma density, $\bar{n}_{e}$, precisely monotonically decreasing at low $\bar{n}_{e}$ (low-density branch) and monotonically increasing at medium-high $\bar{n}_{e}$ (high-density branch) $[2,3,4]$. Immediately after the $\mathrm{H}$-mode discovery [1], a correlation between sawtooth crash and L-H transition was observed [5]: A heat wave which propagates towards the plasma edge is associated with the sawtooth crash, and because of the correlation between sawtooth crash and the formation of the ETB, the heat wave was thought to be the cause of the ETB formation [5]. At that time, however, it was not clear whether this heat wave was carried by all the plasma species, or only by one and which [6]. It became clear that ion-heat flux was the real responsible for the creation of the ETB, only when preciser measurements of the ion temperature were available and thanks to dedicated experiments with dominant ion heating, e.g. low-voltage neutral-beam-injection (NBI) heating [7] and ${ }^{3} \mathrm{He}$-minority ion-cyclotron resonance heating [8], in comparison to heating schemes with more electron heating $[3,7]$. If ions are the vector of the heat flux for the L-H transition, the non-monotonic behaviour of $P_{\mathrm{L}-\mathrm{H}}$ is naturally connected to the energy equipartition between electrons and ions, which decreases with the plasma density. This energy equipartition is particularly relevant in plasma with dominant electron heating $[3,7]$. The density of the $P_{\mathrm{L}-\mathrm{H}}$ minimum and its dependence on the plasma parameters are still subject of research, although in the years a number of models of various complexity have been proposed for the L-H transition [9]. Here, we recall only three of the more recent models capable to capture the $\mathrm{P}_{\mathrm{L}-\mathrm{H}}$ minimum and not described in [9]. Chronologically, the first model [10] is based on the physical hypothesis that L-H transition appears when nonlinear drift waves transfer energy to the zonal flows via inverse energy cascade, which in turn contribute to $E \times B$ velocity shear, eventually responsible for the turbulence quench. In the second model [11], the existence of the $\mathrm{P}_{\mathrm{LH}}$ minimum is attributed to the fact that at low plasma densities the convection contribution to the turbulent power through the edge-dissipation layer overwhelms the conduction part. However, none of these two models entail a clear distinction between the ion and electron channels, as observed experimentally $[3,7]$. Finally, the third model [12] introduces this dependence explicitly, and the $P_{\mathrm{L}-\mathrm{H}}$ increase with the density in the high-density branch is captured by an increase of the ion-collisional damping of the turbulence-driven shear flows.

In this Letter, we propose a phenomenological model for $P_{\mathrm{L}-\mathrm{H}}$ which captures the $P_{\mathrm{L}-\mathrm{H}}$ minimum, and describes the dependencies of $P_{\mathrm{L}-\mathrm{H}}$ on the main plasma parameters. It is based mainly on three experimental observations. We have already mentioned the first, namely the key role of the ion heat flux. The second important experimental finding is the dependence of the ion-power threshold for the $\mathrm{L}-\mathrm{H}$ transition, $P_{\mathrm{L}-\mathrm{H}, \mathrm{i}}$, on the plasma density, magnetic field, and plasma surface, described by the AUG-C-Mod scaling [7, 8, 13],

$$
P_{\mathrm{L}-\mathrm{H}, i}=0.0029 \bar{n}_{e}^{1.05 \pm 0.1} B^{0.68 \pm 0.3} S^{0.93 \pm 0.2} .
$$


with $\bar{n}_{e}$ in $10^{19} \mathrm{~m}^{-3}, B$ the toroidal magnetic field in Tesla, and $S$ the plasma surface area in $\mathrm{m}^{2}$. The third observation, relevant for the present analysis, is that $P_{\mathrm{L}-\mathrm{H}}$ has a minimum at a plasma density such that the ratio between the energy confinement time, $\tau_{\mathrm{th}}$ and the electron-ion energy equipartition time, $\tau_{\mathrm{eq}}^{e / i}[7]$ takes a specific value. The expression of the density at the $P_{\mathrm{L}-\mathrm{H}}$ minimum obtained from this constraint is rather universal and verified on an extended set of discharges from various fusion devices with different wall materials [7].

In the next section we use these three observations to build the phenomenological model and show its capability to reproduce the experimental data [3]. However, since this model is not fully derived from first principles, it unavoidably depends on numerical constants that follow from (1) and from the L-mode scaling for the energy confinement time (see later). In Section 3 we substantially expand an argument to derive from first principles the $B, \bar{n}_{e}$, and $S$ dependence's in (1). This argument was originally proposed by F. Wagner and documented in section 1 of the review paper [9]. In the last section we draw some conclusions.

\section{Phenomenological model for the L-H power threshold}

We first consider the definition of the global energy confinement time [14]

$$
\tau_{\mathrm{th}}=\frac{W_{\mathrm{th}}}{P_{\mathrm{loss}}}=\frac{3}{2} \frac{\left\langle n_{e} T_{e}\right\rangle_{V}+\left\langle n_{i} T_{i}\right\rangle_{V}}{P_{\mathrm{loss}}} V
$$

(we assume that $n_{i}=n_{e}$, as it is the case for the typical plasmas dominated by hydrogen isotopes) where $W_{\text {th }}$ is the total internal plasma energy, $\langle A\rangle_{V}:=V^{-1} \int A \mathrm{~d} V$ stands for the volume average, and $P_{\text {loss }}$ is defined as [15]

$$
P_{\text {loss }}:=P_{\text {aux }}+I_{P} V_{\text {loop }}-\frac{\mathrm{d} W_{\mathrm{th}}}{\mathrm{d} t},
$$

where: $V_{\text {loop }}$ is the loop voltage at the plasma edge, $I_{p}$ is the toroidal plasma current, and in steady-state $\left(I_{P} V_{\text {loop }}\right)$ is the Ohmic heating; $P_{\text {aux }}$ the total auxiliary plasma heating. With the definition of the density-weighted average, $\langle A\rangle_{n_{e}}:=\left\langle n_{e} A\right\rangle_{V} /\left\langle n_{e}\right\rangle_{V}$ the energy confinement time becomes, $\tau_{\text {th }}=(3 / 2)\left\langle n_{e}\right\rangle_{V}\left(\left\langle T_{e}\right\rangle_{n_{e}}+\left\langle T_{i}\right\rangle_{n_{e}}\right) V / P_{\text {loss }}$. The key ansatz in this Letter is to explicitly separate the ion and electron contributions to $\tau_{\text {th }}$, since only ions are the real responsible for the ETB [7]. However, defining the ion- and electron-energy confinement times separately might be ambiguous when electrons and ions have different radial temperature profiles because of the role played by the energy equipartition. If we observe that the energy going from the hotter to the colder species via energy equipartition is confined according to the characteristic energy confinement time of the colder species, this should settle the issue as

$$
\tau_{\mathrm{th}, i}:=\frac{3}{2} \frac{\left\langle n_{e}\right\rangle_{V}\left\langle T_{i}\right\rangle_{n_{e}} V}{\tilde{P}_{\mathrm{loss}, i}}, \quad \text { with }: \quad \tilde{P}_{\mathrm{loss}, i}=P_{\mathrm{loss}, i}+P_{\mathrm{eq}}^{e / i},
$$

with an analogous definition for $\tau_{\mathrm{th}, e} . P_{\text {loss }, \mathrm{i}}$ is the ion heat flux at the separatrix without the energy-equipartition contribution, whereas the power exchange according to the energy equipartition, $P_{\mathrm{eq}}^{i / e}$, is

$$
P_{\mathrm{eq}}^{e / i}:=\frac{3}{2}\left\langle\frac{n_{e}\left(T_{e}-T_{i}\right)}{\tau_{\mathrm{eq}}^{e / i}}\right\rangle_{V} V \approx \frac{3}{2}\left\langle n_{e}\right\rangle_{V} \frac{\left\langle T_{e}\right\rangle_{n_{e}}-\left\langle T_{i}\right\rangle_{n_{e}}}{\left\langle\tau_{\mathrm{eq}}^{e / i}\right\rangle_{V}} V=\tilde{P}_{\mathrm{loss}, i} \frac{\tau_{\mathrm{th}, i}}{\left\langle\tau_{\mathrm{eq}}^{e / i}\right\rangle_{V}} \frac{\langle\Delta T\rangle_{n_{e}}}{\left\langle T_{i}\right\rangle_{n_{e}}},
$$


(note $P_{\mathrm{eq}}^{e / i}=-P_{\mathrm{eq}}^{i / e}$ ), which defines $\langle\Delta T\rangle_{n_{e}} /\left\langle T_{i}\right\rangle_{n_{e}}$

$$
\frac{\langle\Delta T\rangle_{n_{e}}}{\left\langle T_{i}\right\rangle_{n_{e}}} \approx \frac{\left\langle\tau_{\mathrm{eq}}^{e / i}\right\rangle_{V}}{\tau_{\mathrm{th}, i}} \frac{P_{\mathrm{eq}}^{e / i}}{\tilde{P}_{\mathrm{loss}, i}}=\frac{\left\langle\tau_{\mathrm{eq}}^{e / i}\right\rangle_{V}}{\tau_{\mathrm{th}, i}} \Pi_{\mathrm{eq}}^{e / i}, \quad \text { with }: \quad \Pi_{\mathrm{eq}}^{e / i}:=\frac{P_{\mathrm{eq}}^{e / i}}{\tilde{P}_{\mathrm{loss}, i}} .
$$

In the case of dominant electron heating with electron cyclotron resonance heating (ECRH) the parameter $\Pi_{\mathrm{eq}}^{e / i}$ is approximately one. AUG-C-Mod scaling (1) holds for $\tilde{P}_{\text {loss }, i}$ (i.e. accounting also for the energy-equipartition contribution), but it is $P_{\text {loss }}$ that usually enters the scaling laws of the power threshold of the L-H transition [16]. Thus, the global $P_{\text {loss }}$ must be expressed in terms of $\tilde{P}_{\text {loss }, i}$

$$
P_{\mathrm{loss}}=\tilde{P}_{\mathrm{loss}, e}+\tilde{P}_{\mathrm{loss}, i}=\left[\left(1+\frac{\tau_{\mathrm{th}, i}}{\tau_{\mathrm{th}, e}}\right)+\frac{\tau_{\mathrm{th}}}{\tau_{\mathrm{th}, e}} \Pi_{\mathrm{eq}}^{e / i} \frac{\left\langle\tau_{\mathrm{eq}}^{e / i}\right\rangle_{V}}{\tau_{\mathrm{th}}}\right] \tilde{P}_{\mathrm{loss}, i} .
$$

Upon using in (6) the fact that the $\mathrm{L}-\mathrm{H}$ transition occurs when $\tilde{P}_{\text {loss }, \mathrm{i}} \gtrsim P_{\mathrm{L}-\mathrm{H}, \mathrm{i}}$, the criterion on $P_{\mathrm{L}-\mathrm{H}}$ for the $\mathrm{L}-\mathrm{H}$ transition becomes

$$
P_{\text {loss }} \gtrsim P_{\mathrm{L}-\mathrm{H}}:=\left[\left(1+\frac{\tau_{\mathrm{th}, i}}{\tau_{\mathrm{th}, e}}\right)+\frac{\tau_{\mathrm{th}}}{\tau_{\mathrm{th}, e}} \Pi_{\mathrm{eq}}^{e / i} \frac{\left\langle\tau_{\mathrm{eq}}^{e / i}\right\rangle_{V}}{\tau_{\mathrm{th}}}\right] P_{\mathrm{L}-\mathrm{H}, \mathrm{i}} .
$$

In the case of dominant ion heating, $P_{\text {loss }, \mathrm{i}} \gg P_{\mathrm{eq}}^{e / i}\left(\right.$ i.e. $\Pi_{\mathrm{eq}}^{e / i} \approx 0$ ), the power threshold for the $\mathrm{L}-\mathrm{H}$ transition, $P_{\mathrm{L}-\mathrm{H}}$, differs from the ion power threshold $P_{\mathrm{L}-\mathrm{H}, \mathrm{i}}$ only by the electron part $\left(\propto \tau_{\mathrm{th}, i} / \tau_{\mathrm{th}, e}\right)$, which does not contribute to heat flux responsible for the L-H transition [5]. When the energy confinement time is much shorter than the energy equipartition time, $P_{\text {loss }}$ diverges, since the fraction of power transferred from electrons to ions due the energy-equipartition mechanism decreases with $\tau_{\text {eq }}^{e / i} / \tau_{\text {th }}$. To reduce the number of unknowns in (7), for $\tau_{\text {eq }}^{e / i}$ we use the definition of the local energy equipartition time [17],

$$
\left\langle\tau_{\mathrm{eq}}^{e / i}\right\rangle_{V}:=\frac{1}{2} \frac{m_{i}}{m_{e}}\left\langle\tau_{\mathrm{coll}}^{e / i}\right\rangle_{V} \approx 2 \cdot 10^{-6} \frac{A_{i}}{Z_{i}}\left\langle\frac{T_{e}^{3 / 2}}{n_{e}}\right\rangle_{V} \approx 2 \cdot 10^{-6} \frac{A_{i}}{Z_{i}} \frac{\left\langle T_{e}^{3 / 2}\right\rangle_{V}}{\left\langle n_{e}\right\rangle_{V}},
$$

with $\tau_{\text {coll }}^{e / i}$ the electron-ion collisional characteristic time, the electron temperature, $T_{e}$, in $\mathrm{eV}$ and $n_{e}$ in $10^{19} \mathrm{~m}^{-3}$. For $\tau_{\text {th }}$ we apply the experimental scaling for L-mode plasmas (equation (7) in [18])

$$
\begin{aligned}
& \tau_{\mathrm{th}}=\tilde{\tau}_{\mathrm{th}} \bar{n}_{e}^{\nu_{\tau}} P_{\mathrm{loss}}^{\pi_{\tau}}, \quad \text { with }: \quad \nu_{\tau}=0.4, \pi_{\tau}=-0.73, \\
& \tilde{\tau}_{\mathrm{th}}:=0.023 I_{p}^{0.96} B_{T}^{0.03} \kappa^{0.64} R^{1.83}\left(\frac{R}{a}\right)^{0.06} A_{\mathrm{eff}}^{0.20},
\end{aligned}
$$

in units of seconds, megamps, metres, tesla, atomic mass and megawatts. In pursuing the reduction of the number of free parameters, the L-scaling of $\tau_{\text {th }}$ is also used to express $T_{e}$ as function of $P_{\text {loss }}$ in (8) by applying the definition (2). However, strictly speaking this procedure gives the average plasma temperature, $T$, but in plasmas with dominant electron cyclotron heating (ECRH), especially in low-density plasmas, it holds $T_{e} \geq T$. We formally account for this in the model by writing $\left\langle\tau_{\mathrm{eq}}^{e / i}\right\rangle_{V}=\left.\alpha_{\tau}\left\langle\tau_{\mathrm{eq}}^{e / i}\right\rangle_{V}\right|_{T_{e}=T}$ with $\alpha_{\tau}:=\left[1+\langle\Delta T\rangle /\left\langle T_{e}\right\rangle / 2\right]^{3 / 2}$ typically larger than one, 
and

$\left.\frac{\left\langle\tau_{\mathrm{eq}}^{e / i}\right\rangle_{V}}{\tau_{\mathrm{th}}}\right|_{T_{e}=T}=\mathcal{T} \frac{P_{\mathrm{loss}}^{3 / 2+\pi_{\tau} / 2}}{\bar{n}_{e}^{5 / 2-\nu_{\tau} / 2}}, \mathcal{T}:=2 \cdot 10^{-6} \frac{A_{i}}{Z_{i}}\left(\frac{1}{3}\right)^{3 / 2}\left(\frac{10^{6}}{1.6022}\right)^{3 / 2} \frac{P_{\mathrm{loss}}^{3 / 2}}{\bar{n}_{e}^{5 / 2} V^{3 / 2}} \tau_{\mathrm{th}}^{1 / 2}$.

Moreover, the experimental scalings are usually done with the measured line-averaged plasma density, $\bar{n}_{e}$, corresponding to a line of sight passing close to the magnetic axis, and similarly for the temperature. However, the volume averages of the density and temperature enter the definition of $\tau_{\text {eq }}^{e / i}$ and $\tau_{\text {th }}$. This introduces an additional correction parameter $\beta_{\tau}$. Eventually, the criterion (7) becomes an implicit relation for $P_{\mathrm{L}-\mathrm{H}}$

$$
P_{\mathrm{L}-\mathrm{H}}=\left(1+\frac{\tau_{\mathrm{th}, i}}{\tau_{\mathrm{th}, e}}+\Xi^{e / i} \mathcal{T} \frac{P_{\mathrm{L}-\mathrm{H}}^{3 / 2+\pi_{\tau} / 2}}{\bar{n}_{e}^{5 / 2-\nu_{\tau} / 2}}\right) P_{\mathrm{L}-\mathrm{H}, i} .
$$

where for compactness we introduce $\Xi^{e / i}:=\alpha_{\tau} \beta_{\tau}\left(\tau_{\mathrm{th}} / \tau_{\mathrm{th}, e}\right) \Pi_{\mathrm{eq}}^{e / i}$. Since in present fusion devices $P_{\mathrm{L}-\mathrm{H}}$ is typically a few $\mathrm{MW}$, the exponent of $P_{\mathrm{L}-\mathrm{H}}$ on the rhs is approximated with $3 / 2+\pi_{\tau} / 2 \approx 1$ to explicit (11),

$$
P_{\mathrm{L}-\mathrm{H}} \approx\left(1-\frac{\Xi^{e / i} \mathcal{T}}{\bar{n}_{e}^{5 / 2-\nu_{\tau} / 2}} P_{\mathrm{L}-\mathrm{H}, i}\right)^{-1}\left(1+\frac{\tau_{\mathrm{th}, i}}{\tau_{\mathrm{th}, e}}\right) P_{\mathrm{L}-\mathrm{H}, i}
$$

This is the key result of the present model. Since all the parameters in (12) are positive, except for $\Pi_{\text {eq }}^{e / i}$ in $\Xi^{e / i}, P_{\mathrm{L}-\mathrm{H}}$ admits a minimum as function of the density only when $\Pi_{\mathrm{eq}}^{e / i} \gtrsim 0$. Reformulating it in the other way around, according to (12) only in the case of external dominant ion heating, i.e. $T_{i} \gtrsim T_{e}, P_{\mathrm{L}-\mathrm{H}}$ does not have a minimum, as experimentally shown with dedicated experiments at low-voltage NBI heating [7]. Additionally, in the high-density branch $P_{\mathrm{L}-\mathrm{H}} \approx\left(1+\tau_{\mathrm{th}, i} / \tau_{\mathrm{th}, e}\right) P_{\mathrm{L}-\mathrm{H}, i} \gtrsim P_{\mathrm{L}-\mathrm{H}, i}$, which strengthens further that the fraction of the power transported as electron heat flux without being eventually collisionally transferred to the ions enters as a "loss" term in the computation of the power necessary for the $\mathrm{L}-\mathrm{H}$ transition, and thus increases $P_{\mathrm{L}-\mathrm{H}}$. The expression of the density at the minimum of $P_{\mathrm{L}-\mathrm{H}}$ follows immediately from (12), if we explicit the density dependence of $P_{\mathrm{L}-\mathrm{H}, i}$ in (1), i.e. $P_{\mathrm{L}-\mathrm{H}, i}=\tilde{P}_{\mathrm{L}-\mathrm{H}, i} \bar{n}_{e}^{\nu_{P}}$, with $\nu_{p} \approx 1$ from (1), and neglect a possible density dependence in $\tau_{\text {th,i }} / \tau_{\text {th,e }}$

$$
\left.\bar{n}_{e}^{5 / 2-\nu_{\tau} / 2-\nu_{P}}\right|_{\min \left(P_{\mathrm{L}-\mathrm{H}}\right)}=\Xi^{e / i} \mathcal{T} \tilde{P}_{\mathrm{L}-\mathrm{H}, i} \frac{5-\nu_{\tau}}{2 \nu_{P}},
$$

which gives

$$
\bar{n}_{e, \min } \approx 0.16\left(\frac{A_{i}}{Z_{i}}\right)^{0.77}\left(\Xi^{e / i}\right)^{0.77} A_{i}^{0.08} B^{0.54} I_{p}^{0.37}\left(\frac{R}{a}\right)^{0.34} a^{-1.22} \frac{\left(1+\kappa^{2}\right)^{0.39}}{\kappa^{0.91}}
$$

where we have approximated the plasma surface and volume with $S \approx$ $(2 \pi R)\left(2 \pi a \sqrt{\left(1+\kappa^{2}\right) / 2}\right), V \approx(2 \pi R)\left(\pi a^{2} \kappa\right)$, with $a$ and $R$ the minor and major radii, respectively. The expression (14) is close to the experimental result for deuterium plasmas, equation (3) in [7], which we re-write by keeping explicit the dependence on the plasma elongation, $\kappa$,

$$
\bar{n}_{e, \min }^{\mathrm{Ryter}} \approx 0.68 B^{0.62} I_{p}^{0.32}\left(\frac{R}{a}\right)^{0.35} a^{-0.97} \frac{\left(1+\kappa^{2}\right)^{0.36}}{\kappa^{0.8}} .
$$


In ASDEX Upgrade it was observed that in hydrogen, deuterium and ${ }^{4} \mathrm{He}$ plasmas $\bar{n}_{e, \text { min }}$ was very weakly dependent on the $A_{i}$ and $Z_{i}$ [3]. Since the explicit isotope dependence of $\bar{n}_{e, \min }$ in (14) is mainly due to the presence of $A_{i} / Z_{i}$ in $\mathcal{T}$ from the equipartition time, a natural way to incorporate this experimental finding [3] in (13) is to assume that $P_{\mathrm{L}-\mathrm{H}, \mathrm{i}} \propto Z_{i} / A_{i}$. Indeed, this has been seen in deuterium and hydrogen plasmas [19], which corroborates at least $P_{\mathrm{L}-\mathrm{H}, \mathrm{i}} \propto A_{i}^{-1}$. The derivation of $A_{i}, Z_{i}$-dependence of $P_{\mathrm{L}-\mathrm{H}, \mathrm{i}}$ from first principles is still elusive, and here we take $P_{\mathrm{L}-\mathrm{H}, \mathrm{i}} \propto Z_{i} / A_{i}$ as an experimental educated hypothesis, and precisely multiply (1) by $\left(A_{D} / Z_{D}\right) Z_{i} / A_{i}$. In (14) this translated in replacing $\left(A_{i} / Z_{i}\right)^{0.77}$ with $\left(A_{D} / Z_{D}\right)^{0.77} \approx$ 1.7. We will see at the end of this section that this hypothesis rather well extends equation (1) to hydrogen and ${ }^{4} \mathrm{He}$ plasmas, although (1) was derived from a dataset of deuterium discharges [8]. The residual dependence on $A_{i}$ in (14) due to $\tau_{\text {th }}$, namely $A_{i}^{0.08}$, is negligible. The numerical coefficients in (14) and (15) are equal if $\Xi^{e / i} \approx 4.5$ for $\kappa \approx 1.6$. As a matter of fact, expression (12) only has two unknowns, namely $\tau_{\mathrm{th}, i} / \tau_{\mathrm{th}, e}$ and $\Xi^{e / i}$, and in the absence of their experimental scalings, as in the case of $\tau_{\text {th }}$, they can be considered as free fitting parameters.
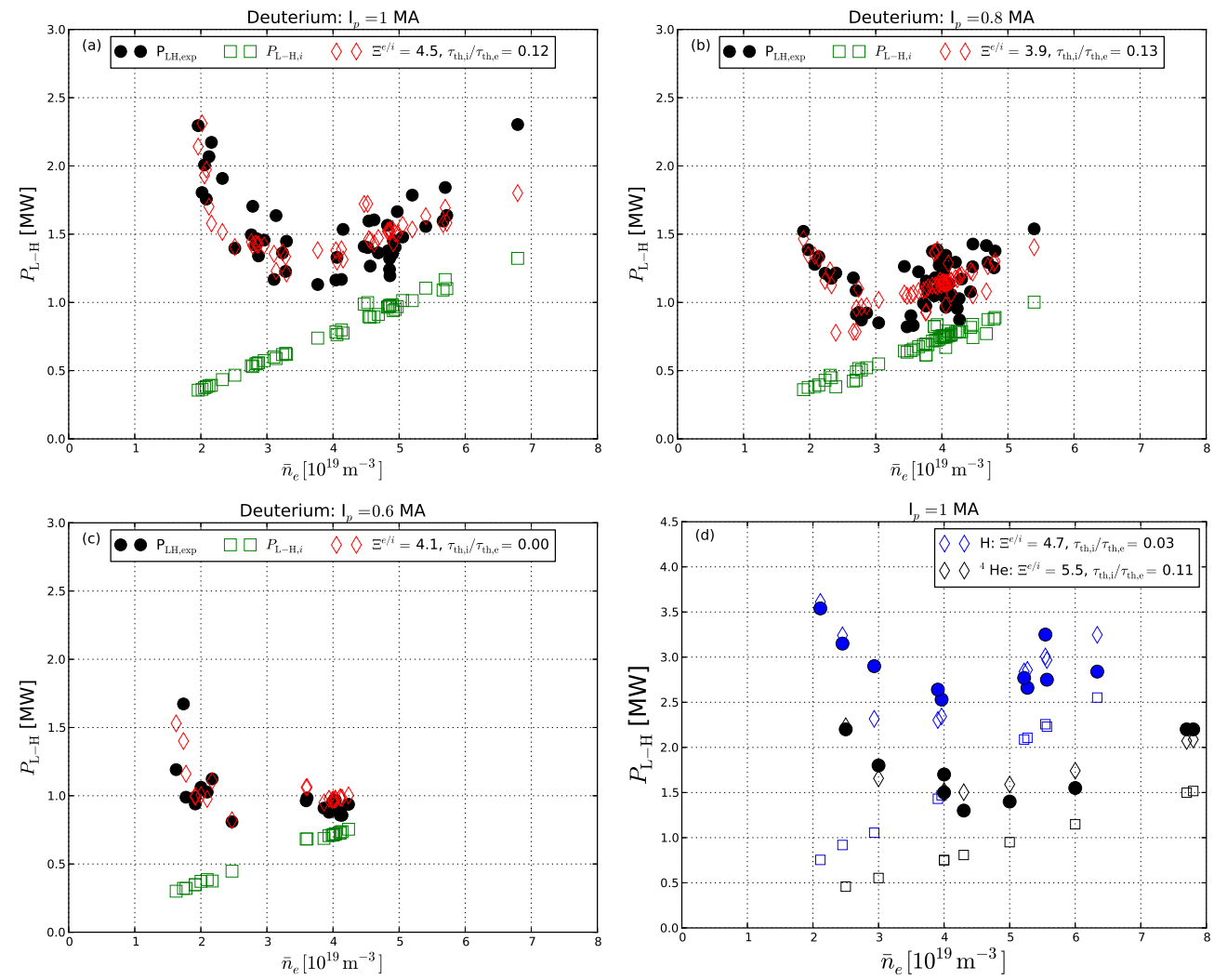

Figure 1. Full bullets are the experimental values of $P_{\mathrm{L}-\mathrm{H}}$ as function of the line-averaged density from the ASDEX Upgrade database of $[3,7]$. The open squares are the values of $P_{\mathrm{L}-\mathrm{H}, \mathrm{i}}$ according to (1). The open diamonds are the best fit with the model (12), and the corresponding $\Xi^{e / i}$ and $\tau_{\mathrm{th}, \mathrm{i}} / \tau_{\mathrm{th}, \mathrm{e}}$ values are reported in the legend. The frames (a)-(c) refer to deuterium plasmas, whereas (d) to hydrogen (blue) and ${ }^{4} \mathrm{He}$ (black). 
Therefore, we apply (14) as fitting function to the experimental $P_{\mathrm{L}-\mathrm{H}}$ as function of the line-averaged density of the ASDEX Upgrade discharges [3, 7], shown as full bullets in figure 1, for different plasma currents. The open squares in figure 1 are $P_{\mathrm{L}-\mathrm{H}, \mathrm{i}}$ of the AUG-C-Mod scaling (1). The open diamonds are the best fit of (12), with the corresponding values of $\Xi^{e / i}$ and $\tau_{\mathrm{th}, \mathrm{i}} / \tau_{\mathrm{th}, \mathrm{e}}$ reported in the legend. In the specific case of $I_{p} \approx 0.6 \mathrm{MA}$, the experimental data are only in the low density branch and the best fit gives $\tau_{\mathrm{th}, \mathrm{i}} / \tau_{\mathrm{th}, \mathrm{e}}=0$, which has been verified separately also on more recent ASDEX Upgrade plasmas dedicated to L-H transition studies, including those of [20]. This points to the common observation that in discharges with dominant electron heating, $T_{e}>T_{i}$, the ion transport in the core is strongly turbulent with ITG/TEM signature and $\tau_{\text {th,i }} \ll \tau_{\text {th,e }}$. On the other hand, in the high-density branch, captured by the datasets of 0.8 and $1 \mathrm{MA}$ plasma current, electrons heated by ECRH more efficiently heat ions via collisions by reducing $T_{e} / T_{i}$, with a relative improvement of the ion energy confinement $\tau_{\mathrm{th}, \mathrm{i}} / \tau_{\mathrm{th}, \mathrm{e}}>0$. The values of $\Xi^{e / i}$ from the best fits for the three plasma currents are all in the ball-park of what previously obtained by comparing (14) with (15). According to the present model, the dependence on the plasma current in (12) enters through $\tau_{\text {th }}$ of the L-mode scaling, and it has an impact also on the relative distance between $P_{\mathrm{L}-\mathrm{H}}$ and $P_{\mathrm{L}-\mathrm{H}, i}$ at medium and high densities. Finally, in these discharges with ECRH only, $\Pi_{\mathrm{eq}}^{e / i} \approx 1$ and $\left(\tau_{\mathrm{th}} / \tau_{\mathrm{th}, e}\right) \lesssim 1$, and the product $\alpha_{\tau} \beta_{\tau}$, that takes into account differences between $T_{e}$ and $T_{i}$, and differences between volume averaged and line averaged quantities, is indeed about 4 .

We conclude this Section by applying the same model (12) to hydrogen and ${ }^{4} \mathrm{He}$ plasmas, where we have modified $P_{\mathrm{L}-\mathrm{H}, \mathrm{i}}$ of (1), obtained from deuterium plasmas, by multiplying (1) by $\left(A_{D} / Z_{D}\right) Z_{i} / A_{i}$, as previously motivated when discussing the $A_{i} / Z_{i}$-dependence in $n_{e, \min }$, cf. equation (14). The quantitative agreement between the experimental $P_{\mathrm{L}-\mathrm{H}}$ and the model predictions, shown in figure 1.d, is such to make reasonable this extension of (12), in the absence of a first-principle derivation of the numerical coefficient in (1).

\section{Tentative physical picture behind $P_{\mathrm{L}-\mathrm{H}, i}$}

To understand the physics behind the numerical constant in the AUG-C-Mod scaling (1), we use the old observation [5], confirmed and made preciser only recently [7], that a sort of ion-heat flux impinging on the plasma edge from the plasma core is responsible for the L-H transition. We first integrate the ion-heat flux up to the plasma surface where the edge-transport-barrier (ETB) forms at the L-H transition,

$$
Q_{\text {edge }, i}=\int_{V} \nabla \cdot \mathbf{q}_{i} \mathrm{~d} V=\int_{\partial V} \mathbf{q}_{i} \cdot \mathrm{d} \mathbf{S},
$$

with $\partial V$ the plasma boundary, $\mathbf{q}_{i}$ the total (conductive+convective) ion heat flux and $\mathbf{S}$ the plasma surface. According to the steady-state form of the energy-conservation equation [15] it holds $Q_{\text {edge }, i}=\tilde{P}_{\text {loss }, i}$. Then, the ion-temperature gradient, $T_{i}^{\prime}$, is connected to $\tilde{P}_{\text {loss }, i}$ by a steady-state thermal conduction equation in the ETB,

$$
\tilde{P}_{\mathrm{loss}, i} \approx-\hat{\chi}_{i} \hat{n}_{i} \hat{T}^{\prime} \hat{S}=-\hat{\chi}_{i} \frac{\hat{p}_{i}^{\prime}}{1+\hat{\eta}_{i}^{-1}} \hat{S}
$$

where ^stands for the average in the ETB layer, $\hat{\chi}_{i}$ is the characteristic value of the ion-heat diffusivity in the ETB, the prime denotes the radial derivative, and the 
parameter $\eta:=L_{n} / L_{T}$, with $L_{n, T}^{-1}$ the logarithm radial gradients of the density and temperature. Equation (17) is not enough to explain the increase of $\hat{p}_{i}^{\prime}$ observed during the L-H transition when $\tilde{P}_{\text {loss }, i}$ is increased, since in L-mode $\hat{\chi}_{i}$ increases with $\hat{P}_{\text {loss }, i}$ as well $[21,18]$. We need a simultaneous reduction of $\hat{\chi}_{i}$ when $\tilde{P}_{\text {loss }, i}$ is increased. In 1990 Biglari, Diamond, and Terry (BDT) [22, 23] proposed a mechanism, which is still one of the most accredited to explain the L-H transition: Sheared $\mathbf{E} \times \mathbf{B}$ poloidal flows at the plasma edge tear apart turbulence eddies responsible for the low confinement in Lmode. As a consequence of this shear flow, transport due to turbulence is drastically reduced, namely $\hat{\chi}_{i}$ is reduced. Quantitatively, the BDT criterion states that the shearing rate, $\gamma_{E \times B}$, must be larger than the turbulence growth rate, $\gamma_{\text {turb }}$, in order to suppress turbulence in the ETB,

$$
\gamma_{\text {turb }}<\gamma_{E \times B} \approx \frac{R B_{\vartheta}}{B} \frac{\partial}{\partial r}\left(\frac{E_{r}}{R B_{\vartheta}}\right) \approx \frac{1}{\Delta r} \frac{E_{r, \min }}{B},
$$

where $B_{\vartheta}$ is the poloidal component of the confining magnetic field, $B$, and $R$ the distance from the torus axis. In (18), $\gamma_{E \times B}$ is simplified by accounting only for the component dominant in flute-like turbulence [24]. At the plasma edge the dominant gradient is $E_{r}^{\prime}$, and the radial electric field decreases approximately linearly [25, 26]. Thus, $E_{r}^{\prime} \approx \Delta E_{r} / \Delta r \approx E_{r, \min } / \Delta r$, where $\Delta r$ is about the half width of the ETB. The last relation necessary to close the loop between (17) and (18) is the radial force balance equation which connects $E_{r}$ to $\hat{p}_{i}^{\prime}$,

$$
E_{r}=-\frac{\hat{p}_{i}^{\prime}}{Z_{i} e \hat{n}_{i}}-(\mathbf{V} \times \mathbf{B})_{r}+\frac{m_{i}}{Z_{i} e} \frac{\partial}{\partial r}\left\langle\tilde{V}_{i, r} \tilde{V}_{i, \theta}\right\rangle_{n},
$$

with $\mathbf{V}$ the plasma velocity, and with the additional Reynolds-stress contribution (last term on the rhs in (19)), which is claimed to initially reduce $\hat{\chi}_{i}$ to ease the triggering of the $\mathrm{L}-\mathrm{H}$ transition $[27,28]$. In this respect, other mechanisms might be equally important and are not included in (19), such as the non-ambipolar component of the electric field due to non-compensated fast ions losses [9]. These terms might contribute to an initial unbalance in (17) in favour of $\hat{p}_{i}^{\prime}$ and thus might help to initiate the beneficial loop, $\tilde{P}_{\text {loss }, i} \longrightarrow \hat{p}_{i}^{\prime} \longrightarrow E_{r} \longrightarrow \hat{\chi}_{i}$ for the ETB creation. The knowledge and, thus, the modeling of these mechanisms are critical when describing the dynamics of the L-H transition [12] (and references cited in [29]). Here, however, we are interested in defining the minimum $\tilde{P}_{\text {loss }, i}$ necessary to achieve a fully developed $\mathrm{H}$-mode, when the third term in (19) is already largely overwhelmed by the diamagnetic contribution (first term on the rhs in (19)). A separate comment deserves the $\mathbf{V} \times \mathbf{B}$ contribution, relevant in rotating plasmas. This can indeed impact the power threshold for the $\mathrm{L}-\mathrm{H}$ transition by either increasing or decreasing it depending on the direction of the plasma rotation [30]. In the following, we neglect this term as well, and derive considerations valid especially for discharges with dominant auxiliary wave heating. If we use (19) without the second and third terms to express $\hat{p}_{i}^{\prime}$ as function of $E_{r}$, equation (17) becomes

$$
\tilde{P}_{\text {loss }, i} \approx Z_{i} e \frac{\hat{\chi}_{i}}{1+\hat{\eta}_{i}^{-1}} \hat{E}_{r} \hat{n}_{i} \hat{S}
$$

and the BTD criterion (18) translates into a constraint on $\tilde{P}_{\text {loss }, i}$,

$$
\tilde{P}_{\text {loss }, i} \gtrsim P_{\mathrm{L}-\mathrm{H}, i}:=\left[e \frac{\Delta r \gamma_{\mathrm{turb}} \hat{\chi}_{i}}{1+\hat{\eta}_{i}^{-1}}\right] B \hat{n}_{e} \hat{S},
$$


with $n_{e}=Z_{i} n_{i}$. The strong analogy between (21) and the AUG-C-Mod scaling [7, $8]$ is certainly intriguing. However, before asserting that (21) is confirmed by the experimental scaling (1), or alternatively that (21) explains the experimental scaling (1), it is necessary to investigate whether further important dependencies on $n_{e}$ and on $B$ are hidden in $\left(\gamma_{\text {turb }} \Delta r \hat{\chi}_{i}\right)$. Here, we just propose an incomplete and qualitative argument, mainly based on experimental facts. Experimentally, $\Delta r$ seems not to strongly depend either on $n_{e}$ or on $B$ [31], and only to slightly depend on the isotopic mass [32, 33, 34]. A recent analysis of $E_{r}$ measurements in the ETB on AUG shows that $E_{r, \min }$ increases linearly with $B$, and thus $E_{r, \min } /(\Delta r B)$ does not strongly depend on $B$, as well as not on the density and isotopic mass [13]. This experimental fact together with the BDT criterion (18) and the previous remarks on $\Delta r$ imply that at the L-H transition $\gamma_{\text {turb }}$ does not depend sensitively either on $n_{e}$ or on $B$. The last term left is $\hat{\chi}_{i}$, which indeed can depend on $B$. In fact, both Bohm and gyro-Bohm scalings for $\hat{\chi}_{i}$ predict a power dependence on $B$, namely $B^{-\alpha}$ with $\alpha=-1,-2$, respectively [35], and, if applied to (21), both these dependencies over-compensate the $B$ dependence already present in (21). However, all the known L-mode scalings of the energy confinement time show a very weak dependence on $B[21,18]$, and this suggests that in L-mode $\chi_{i}$ has a weak dependence on $B$, especially in the periphery where the largest plasma volume is present. This hypothesis is compatible with the fact that the exponent of $B$ in the experimental scaling (1) is smaller than one, but still definitively larger than zero. This qualitative argument, based on experimental observations, substantiates the close relation between the experimental scaling (1) and the physical picture behind (21).

Definitively more challenging is to justify why $P_{\mathrm{L}-\mathrm{H}, i}$, derived for deuterium plasmas [8], multiplied by $\left(A_{D} / A_{i}\right)\left(Z_{i} / Z_{D}\right)$ reproduces also $P_{\mathrm{L}-\mathrm{H}}$ in hydrogen and ${ }^{4} \mathrm{He}$ plasmas, as shown in figure 1.d. A simplified argument to explain the isotopic mass dependence, i.e. $A_{D} / A_{i}$, of the factor that multiplies $P_{\mathrm{L}-\mathrm{H}, i}$, is the following. Since ions play the dominant role in the L-H transition, we assume that the ion-branch, characterized by $k_{y} \rho_{i} \ll 1\left(\rho_{i}=v_{\text {thi }} / \Omega_{i}\right.$ is the ion Larmor radius, $\left.\Omega_{i}=Z_{i} e B /\left(m_{i} c\right)\right)$, dominates the turbulence transport at the L-H transition. The self-similarity of turbulence spectra in $k_{y} \rho_{i}$, for $k_{y} \rho_{i} \ll 1$, is such that $\gamma_{\text {turb }} \propto \operatorname{cst} c_{s} / \sqrt{R L_{T}}\left(k_{y} \rho_{i}\right)$ (with $c_{s}=\sqrt{T_{e} / m_{i}}$ the sound speed) and $\gamma_{\text {turb }}$ peaks around $k_{y} \rho_{i} \approx 0.3[36,37]$. This implies that $\gamma_{\text {turb }} \propto A_{i}^{-1 / 2}$. To proceed we observe that in a local-quasilinearelectrostatic approach $\hat{\chi}_{i} \approx \gamma_{\text {turb }} / k_{x}^{2}$ with $k_{x}^{-1}$ the characteristic length of the radial inhomogeneity [38]. Typically, $k_{x} \rho_{s} \ll 1$ [39], and under the hypothesis that where the ETB forms $k_{x}^{-1}$ does not scale with $\rho_{s}$, we obtain that $\hat{\chi}_{i} \gamma_{\text {turb }} \approx \gamma_{\text {turb }}^{2} \propto A_{i}^{-1}$, which is the isotopic mass dependence we are looking for. An additional consequence of this unconventional hypothesis is that $\tau_{\text {th }} \approx \hat{\chi}_{i}^{-1} \propto A_{i}^{0.5}$, close to the experimental scaling $A_{i}^{0.2}$ (i.e. the so-called isotope-effect in the energy confinement) [18, 40]. Finding a simple possible explanation for the second factor $Z_{i} / Z_{D}$ is definitively much more challenging, but with much less practical impact, since as fusion fuel in a reactor we are mainly interested in the isotopes of hydrogen, i.e. $Z_{i} / Z_{D}=1$.

Finally, the impact of the density gradient in lowering the power threshold for the $\mathrm{L}-\mathrm{H}$ transition is represented by $\hat{\eta}_{i}^{-1}$ in (21): this might account for the reduction of the L-H power threshold when replacing carbon by tungsten wall in AUG, where an increase of the density gradient is indeed measured [41, 42]. Similarly, the favourable $\mathbf{B} \times \nabla \mathbf{B}$ direction towards the divertor might contribute to increase $\hat{\eta}_{i}$ at the edge, and thus to reduce the L-H power threshold, as observed experimentally [43]. However, 
$\hat{\eta}_{i}$ together with the other parameters in bracket in (21) depend also on the processes at the plasma edge, largely sensitive to the geometry, material and conditions of the divertor $[44,45]$. In the absence of a quantitative modeling of these parameters, the present model cannot make quantitative predictions for ITER different from those of the ITPA-2008 $P_{\mathrm{L}-\mathrm{H}}$ scaling [16], except for the fact that reducing $\Xi^{e / i}$, and in particular $\Pi_{\mathrm{eq}}^{e / i}$ with dominant ion heating, might beneficially decrease the density of the $P_{\mathrm{L}-\mathrm{H}}$ minimum.

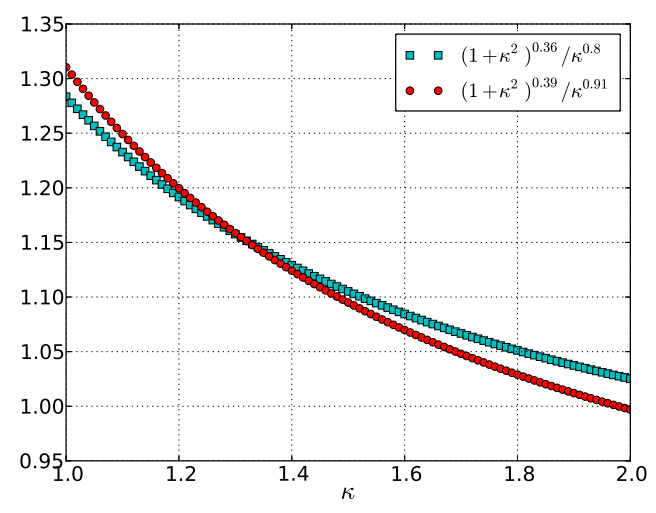

Figure 2. The $\kappa$-dependent factors in (14) and (15) are shown as function of the plasma elongation, $\kappa$.

\section{Conclusions}

In summary, despite its simplicity, this phenomenological model, based on experimental findings and inspired by some very general theoretical concepts, describes the transition of $P_{\mathrm{L}-\mathrm{H}}$ from low- to high-density branch, passing through the minimum, in good agreement with experimental trends [7]. It might offer guidelines in more sophisticated and quantitative modeling of the L-H transition, which are unavoidably based on complex transport numerical tools. This model sheds light on the dependence of the power threshold on the plasma current, which is strong when the energy equipartition time between electrons and ions is long enough to be comparable to the energy confinement time. The power threshold $P_{\mathrm{L}-\mathrm{H}}$ cannot be lower than $P_{\mathrm{L}-\mathrm{H}, i}$ (equations (21) and (1)), necessary condition to reduce turbulence at the edge and create an ETB according to the BDT criterion (18). The density below which the power threshold $P_{\mathrm{L}-\mathrm{H}}$ starts to increase and departs from $P_{\mathrm{L}-\mathrm{H}, i}$ depends on $B, I_{p}$, and on the plasma elongation, $\kappa$. One way to reduce $n_{e, \text { min }}$ by keeping the same $B$ and $I_{p}$ is to increase the plasma elongation. The impact of the $\kappa$-dependence of $P_{\mathrm{L}-\mathrm{H}}$ in (14) and (15) is shown in figure (2): by moving from a circular to a strong elongated plasma, the density of the $P_{\mathrm{L}-\mathrm{H}}$ minimum can decrease by about $20 \%$.

Acknowledgments: We would like to thank M. Cavedon, A. Chankin, E. Fable, U. Plank, E. Poli, and H. Zohm for the fruitful discussions. R. B. is grateful to F. Wagner for the interesting discussions and the careful reading of the manuscript. We would like to thank the anonymous reviewers for their helpful comments and suggestions. 
This work has been partly carried out within the framework of the EUROfusion Consortium and has received funding from the Euratom research and training programme 2014-2018 and 2019-2020 under grant agreement number 633053. The views and opinions expressed herein do not necessarily reflect those of the European Commission.

\section{References}

[1] WAGneR, F., BECKER, G., BEHRINGER, K., et al., Phys. Rev. Lett. 49 (1982) 1408.

[2] SNIPES, J., GRANETZ, R., GREENWALD, M., et al., Nuclear Fusion 36 (1996) 1217.

[3] RYTER, F., RATHGEBER, S., ORTE, L. B., et al., Nuclear Fusion 53 (2013) 113003.

[4] MAGGI, C., Delabie, E., BIEWER, T., et al., Nuclear Fusion 54 (2014) 023007.

[5] WAGneR, F., FUSSMAnN, G., GRAVE, T., et al., Phys. Rev. Lett. 53 (1984) 1453.

[6] WAGNER, F., The European Physical Journal H 43 (2018) 523.

[7] RYTER, F., ORTE, L. B., KURZAN, B., et al., Nuclear Fusion 54 (2014) 083003.

[8] SCHMIDTMAYR, M., HUGHES, J., RYTER, F., et al., Nuclear Fusion 58 (2018) 056003.

[9] CONnOR, J. W. and WILSON, H. R., Plasma Physics and Controlled Fusion 42 (2000) R1.

[10] FUNDAMENSKI, W., MiLITELlO, F., MOULTON, D., and MCDONALD, D., Nuclear Fusion 52 (2012) 062003.

[11] SINGH, R., JHANG, H., KAW, P. K., et al., Physics of Plasmas 21 (2014) 062503.

[12] MAlKOV, M. A., DIAMOND, P. H., MIKI, K., RICE, J. E., and TYNAN, G. R., Physics of Plasmas 22 (2015) 032506.

[13] CAVEDOn, M., BIRKEnMeIER, G., PÜtTERICH, T., et al., Nuclear Fusion 60 (2020) 066026.

[14] WESSON, J., Tokamaks, Clarendon Press, 2003.

[15] HELANDER, P. and SIGMAR, D. J., Collisional Transport in Magnetized Plasmas (Cambridge Monographs on Plasma Physics), Cambridge University Press, 2005.

[16] MARTIN, Y. R., TAKIZUKA, T., and THE ITPA CDBM H-MODE THRESHOLD DATA GROUP, Journal of Physics: Conference Series 123 (2008) 012033.

[17] SPITZER, L., Physics of fully ionized gases, Interscience Publishers, 1956.

[18] KAYE, S., GREENWALD, M., STROTH, U., et al., Nuclear Fusion 37 (1997) 1303.

[19] RYTER, F., CAVEDON, M., HAPPEL, T., et al., Plasma Physics and Controlled Fusion 58 (2016) 014007.

[20] PLANK, U., PÜTTERICH, T., ANGIONI, C., et al., Nuclear Fusion 60 (2020) 074001.

[21] YUSHMANOV, P., TAKIZUKA, T., RIEDEL, K., et al., Nuclear Fusion 30 (1990) 1999.

[22] BIGlari, H., DiAmOnd, P. H., and TERRY, P. W., Physics of Fluids B: Plasma Physics 2 (1990) 1.

[23] TERRY, P. W., Rev. Mod. Phys. 72 (2000) 109.

[24] HAHM, T. S. and BURRELL, K. H., Physics of Plasmas 2 (1995) 1648.

[25] VIEZZER, E., PÜTTERICH, T., CONWAY, G., et al., Nuclear Fusion 53 (2013) 053005.

[26] CAVEDON, M., PÜTTERICH, T., VIEZZER, E., et al., Nuclear Fusion 57 (2017) 014002.

[27] DIAMOND, P. H., LIANG, Y.-M., CARRERAS, B. A., and TERRY, P. W., Phys. Rev. Lett. 72 (1994) 2565.

[28] KIM, E.-J. and DIAMOND, P. H., Phys. Rev. Lett. 90 (2003) 185006.

[29] SCHMITZ, L., Nuclear Fusion 57 (2017) 025003.

[30] GOHIL, P., JERnigAn, T., OSBORNE, T., SCOVIlle, J., and STRAIT, E., Nuclear Fusion 50 (2010) 064011.

[31] SChneider, P., WOlfrum, E., GROEBnER, R., et al., Nuclear Fusion 53 (2013) 073039.

[32] E.J.DOYLE, Nuclear Fusion 47 (2007) S18.

[33] URANO, H., TAKIZUKA, T., KAMADA, Y., OYAMA, N., and AND, H. T., Nuclear Fusion 48 (2008) 045008.

[34] LAgGneR, F. M., WOlfrum, E., CAVEDOn, M., et al., Physics of Plasmas 24 (2017) 056105.

[35] ERBA, M., Cherubini, A., PARAil, V. V., SPRIngmann, E., and TARONi, A., Plasma Physics and Controlled Fusion 39 (1997) 261.

[36] GARCIA, J., GRLER, T., and JENKO, F., Physics of Plasmas 25 (2018) 055902.

[37] IDOMURA, Y., Physics of Plasmas 26 (2019) 120703.

[38] WEILAND, J., Stability and Transport in Magnetic Confinement Systems, Springer, 2012.

[39] BOnAnOMI, N., ANGIONI, C., CRANDAlL, P., et al., Nuclear Fusion 59 (2019) 126025. 
[40] MAgGi, C. F., Weisen, H., HiLlesheim, J. C., et al., Plasma Physics and Controlled Fusion 60 (2017) 014045.

[41] SChNEIDER, P. A., ORTE, L. B., BURCKHART, A., et al., Plasma Physics and Controlled Fusion 57 (2014) 014029.

[42] ShaO, L. M., WOlfrum, E., RYTER, F., et al., Plasma Physics and Controlled Fusion 58 (2016) 025004.

[43] CARLSTROM, T. N., Fusion Science and Technology 48 (2005) 997.

[44] JOFFRIN, E., TAMAIN, P., BELONOHY, E., et al., Nuclear Fusion 57 (2017) 086025.

[45] Chankin, A. V., DElABIE, E., CORRIGAN, G., et al., Plasma Physics and Controlled Fusion 59 (2017) 045012. 